\title{
COLLIDING BLACK HOLES FROM A NULL POINT OF VIEW: THE CLOSE LIMIT
}

\author{
SASCHA HUSA, JEFFREY WINICOUR \\ Department of Physics and Astronomy \\ University of Pittsburgh, Pittsburgh, PA 15260 \\ and \\ Max-Planck-Institut für Gravitationsphysik, Albert-Einstein-Institut \\ 14476 Golm, Germany \\ MANUELA CAMPANELLI \\ Max-Planck-Institut für Gravitationsphysik, Albert-Einstein-Institut \\ 14476 Golm, Germany \\ ROBERTO GOMEZ, YOSEF ZLOCHOWER \\ Department of Physics and Astronomy \\ University of Pittsburgh, Pittsburgh, PA 15260
}

\begin{abstract}
We present a characteristic algorithm for computing the perturbations of a Schwarzschild spacetime by means of solving the Teukolsky equations. Our methods and results are expected to have direct bearing on the study of pinary black holes presently underway using a fully nonlinear characteristic code $\mathrm{U}$.
\end{abstract}

Recent years have shown a vital synergism between numerical and perturbative approaches in the context of Cauchy evolution. In this work we try to make these benefits also accessible in the context of null-surface-based evolution. We treat the "close-limit" approximation to the post-merger phase of a binary black hole spacetime in terms of the characteristicinitial value problem. We outline a framework to treat the Teukolsky equation $\mathrm{a}$ in a null evolution setting, and our numerical strategy to solve the perturbative problem in the time domain. A detailed discussion and results for the outgoing radiation problem are given in 3 , the details of the ingoing radiation problem will be treated in a forthcoming paper 4 .

We apply our method to evolve "close-limit" horizon data, which we obtain from linearizing the data discussed in $\mathrm{O}$. There we use Bondi-Saschs coordinates, which simplify to Israel coordinates in the spherical case, which are based on an affine parameter $u$ along the white hole horizon, normalized to $u=0$ at the bifurcation sphere, and an affine parameter $\lambda$ along the outgoing null cones, fixed to $\lambda=0$ at the white hole horizon and $\left.\left(\partial^{a} u\right)\left(\partial_{a} \lambda\right)=-1\right)$. These coordinates cover the entire Kruskal manifold $r>0$ with remarkably simple analytic behavior, as first discovered by Israel $\mathbf{6}$. Using these convenient coordinates as a starting point, it is straightforward to write the Teukolsky equations explicitly in any background coordinate system.

In order to treat the radiation near $\mathcal{I}^{+}$it is advantageous to consider a "boosted tetrad" $\left(\tilde{l}^{a}, \tilde{n}^{a}, m^{a}, \bar{m}^{a}\right)$ with $\tilde{l}^{a}=-\nabla^{a} \tilde{u}, \tilde{n}^{a}$ satisfying $\tilde{l}^{a} \tilde{n}_{a}=-1$ and a dyad $m^{a}=\frac{1}{\sqrt{2} r}\left(\frac{\partial}{\partial \vartheta}\right)^{a}+\frac{i}{\sin \vartheta}\left(\frac{\partial}{\partial \varphi}\right)^{a}$. We accordingly define boosted Weyl scalars $\tilde{\psi}_{0}=$ $C_{a b c d} \tilde{l}^{a} m^{b} \tilde{l}^{c} m^{d}$ and $\tilde{\psi}_{4}=C_{a b c d} \tilde{n}^{a} \bar{m}^{b} \tilde{n}^{c} \bar{m}^{d}$. This boosted tetrad is adapted to the affine time on $\tilde{u}$ at $\mathcal{I}^{+}$rather than the affine time $u$ at the horizon.

TeukTalk: submitted to World Scientific on December 14, 2018 
This tetrad has proven useful for the evolution of outgoing radiation described by $\tilde{\psi}_{4}$, for ingoing radiation described by $\tilde{\psi}_{0}$, a more sophisticated choice is necessary to achieve optimal results, as will be discussed in 6 .

In order to accurately track the evolution of $\tilde{\psi}_{4}$ well into the power law tail phase (which typically requires more than a $1000 \mathrm{M}$ ) of evolution in Bondi time $\tilde{u}$, we factor out the asymptotic falloff of $\tilde{\psi}_{4}$ for large $r$, we use fixed time steps in Bondi time and a compactified version $\rho$ of the tortoise coordinate $r *=r+2 M \ln (r / 2 M-$ 1), $\rho=\rho_{0} \tan \rho$, where $\rho_{0}$ is an adjustable parameter. The coordiante $\rho$ allows for a fixed grid to accurately resolve the Schwarzschild potential at all times, which proved vital in the tail phase. For a typical choice of initial data the power law tail only sets in after the quasinormal oscillations have decayed by more than 10 orders of magnitude. In order for the final tail not to be lost in machine error it is necessary to evolve the quasinormal phase in quadruple precision.

We have obtained preliminary results to construct close approximation black hole null data without ingoing radiation, by using superposition of solutions for the linear Teukolsky equation, and by utilizing the time symmetry of the background Schwarzschild spacetime which implies a correspondence map of a retarded solution (no incoming radiation) for $\Psi_{\text {f }}$ into an advanced solution (no outgoing radiation) for $\Psi_{0}$. Details will appear in 1 .

\section{Acknowledgments}

M.C. was partially supported by a Marie-Curie Fellowship (HPMF-CT-1999-00334) This work has been partially supported by NSF PHY 9988663 and NSF PHY 9800731 to the University of Pittsburgh. Computer time for this project was provided by the Pittsburgh Supercomputing Center and by NPACI.

\section{References}

1. R. Gomez et. al., Phys. Rev. Lett. 80, 3915-3918 (1998).

2. S. A. Teukolsky, Astrophys. J. 185, 635-647 (1973).

3. Manuela Campanelli, Roberto Gomez, Sascha Husa, Jeffrey Winicour and Yosef Zlochower, gr-qc/0012107.

4. Manuela Campanelli, Roberto Gomez, Sascha Husa, Jeffrey Winicour and Yosef Zlochower, to be published.

5. Roberto Gomez, Sascha Husa and Jeffrey Winicour, gr-qc/0009092.

6. Werner Israel, Phys. Rev. 143, 1016 (1966). 